\begin{tabular}{|c|c|}
\hline Postprint Version & 1.0 \\
\hline Journal website & http://www.sciencedirect.com \\
\hline Pubmed link & $\begin{array}{l}\mathrm{http}: / / \text { www.ncbi.nlm.nih.gov/entrez/query.fcgi? } \mathrm{db}=\text { pubmed\&cmd=Retrieve\&dop } \\
\mathrm{t}=\mathrm{AbstractPlus} \& \text { list_uids=16473503\&query_hl=6\&itool=pubmed_docsum }\end{array}$ \\
\hline DOI & 10.1016/j.ijantimicag.2005.10.015 \\
\hline
\end{tabular}

\title{
Antibiotic treatment and resistance of unselected uropathogens in the elderly
}

\author{
SITA NYS ${ }^{A, *}$, T. VAN MERODE ${ }^{\mathrm{B}}$, A.I.M. BARTELDS ${ }^{\mathrm{C}}$, E.E. STOBBERINGH ${ }^{\mathrm{A}}$

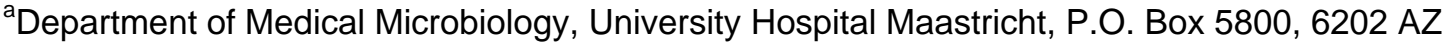 \\ Maastricht, The Netherlands \\ ${ }^{\mathrm{b}}$ Department of General Practice, University of Maastricht, P.O. Box 616, 6200 MD Maastricht, The \\ Netherlands \\ 'The Netherlands Institute for Health Services Research (NIVEL), P.O. Box 1568, 3500 BN Utrecht, \\ The Netherlands \\ *Corresponding author. Tel.: +31 43387 6647; fax: +31 43387 6643. E-mail address: \\ snij@Imib.azm.nl (S. Nys).
}

\begin{abstract}
A population-based study was conducted among women over the age of 70 years with complaints of uncomplicated urinary tract infections (UTIs). The positive predictive value of the nitrite test alone or in combination with the leukocyte esterase test ranged between $83 \%$ and $99 \%$. The nitrofurantoin prescription rate decreased whereas fluoroquinolone and amoxicillin/clavulanic acid prescriptions increased with increasing age. The aetiology of infection was age-dependent. Escherichia coli was the most commonly isolated uropathogen, followed by Proteus mirabilis and Klebsiella pneumoniae. For these uropathogens, the lowest susceptibility percentages were found for amoxicillin, trimethoprim and co-trimoxazole. As trimethoprim susceptibility reached $75 \%$, it may be advisable not to use this as a first-choice agent in the treatment of uncomplicated UTIs in the elderly.
\end{abstract}

\section{INTRODUCTION}

Urinary tract infections (UTIs) are among the most common infections in women and the prevalence increases substantially with increasing age [1], i.e. from $5-10 \%$ for those up to the age of 70 years to $\sim 20 \%$ for those aged 80 years and older [2,3]. Moreover, recurrent UTIs are more prevalent among postmenopausal women than among younger women [4] and these infections are uncomfortable, costly and negatively affect the patient's quality of life [5].

The diagnosis of an acute uncomplicated UTI in older female patients is also more complex than in younger women $[6,7]$ as older women often have an atypical clinical presentation and because many common urinary symptoms (i.e. frequent urination and incontinence) are already present. Furthermore, obtaining a clean-catch (midstream) urine specimen is more difficult [6] and co-morbidities common to the elderly, such as neurological, hepatic and cardiac conditions, can complicate the diagnosis and treatment of a UTI [7]. Moreover, a positive culture, with or without the presence of pyuria, may be an asymptomatic bacteriuria, a condition similar to colonisation that might not always benefit from antibiotic treatment [6,7]. The nitrite and leukocyte esterase dipstick tests have been shown to be reliable diagnostic tools in detecting UTI in young female general practice patients [8-10]. However, no data are available on the performance of either diagnostic test in the elderly community-dwelling female population. 
The aetiology of UTIs among the elderly includes a broad range of Gram-negative and Gram-positive microorganisms. Consequently, these infections require longer treatment duration with broadspectrum antimicrobial agents for optimal treatment. Moreover, the uropathogens isolated in the elderly are more likely to be resistant to conventional antibiotic agents such as trimethoprim, probably owing to selection of more resistant strains by frequent antibiotic use in this population [7,11].

Most antibiotic surveillance studies on UTIs in the elderly female population have focused on institutionalised patients or patients living in long-term care facilities [2,12]. Acute uncomplicated UTIs in older female patients residing (independently) in the community have not been well studied. Therefore, this community-based study of female patients over 70 years of age attending 21 General Practitioners (GPs) from the Sentinel Station of The Netherlands Institute of Health Services Research (NIVEL) with complaints of a UTI was performed. In this surveillance study, the usual care of the GP, i.e. the nitrite test and leukocyte esterase test (if performed), as well as the therapy prescribed were evaluated. Furthermore, the antibiotic susceptibilities of the most prevalent uropathogens were determined.

\section{MATERIALS AND METHODS}

\subsection{Participating GPs}

Twenty-one General Practices from the Sentinel Station of NIVEL participating in the study were located all over The Netherlands. Their patient population accounts for ca. $1 \%$ of the Dutch population and is representative according to age, gender, regional distribution and degree of urbanisation. Female patients over 70 years of age suffering from an acute uncomplicated UTI were included by the GPs from January 2003 until December 2004. The GPs were asked to include all patients with a UTI defined as the presence of one or more of the following complaints: acute signs of dysuria, stranguria, urinary frequency or urgency without the presence of fever $>38^{\circ} \mathrm{C}$. For each patient, age and the usual care performed by the GP (i.e. nitrite and leukocyte esterase tests and the therapy prescribed) were recorded. All patients received care as usual, i.e. diagnostic tests and empirical therapy according to the daily practice of the GP.

\subsection{Urine sampling and processing}

A fresh voided urine sample provided by the patients was used for nitrite dipstick and/or leukocyte esterase test by the GP (when part of the usual care). Subsequently, a dipslide (Uriline, 56508; BioMérieux, Plainview, NY) was prepared according to the manufacturer's instructions and sent to the Laboratory of Medical Microbiology of the University Hospital Maastricht, The Netherlands, for identification and susceptibility testing of the uropathogens.

On the day of arrival, if necessary, the dipslide was incubated at $37^{\circ} \mathrm{C}$ for $16-20 \mathrm{~h}$. Thereafter, growth on the dipslides was compared with a chart provided by the manufacturer and recorded from no growth to $\geq 10^{7}$ colonyforming units (CFU)/mL. Bacterial growth on the dipslides of $\geq 10^{3} \mathrm{CFU} / \mathrm{mL}$ was identified using standard biochemical tests [13]. If there remained any doubt about the identification, further biochemical tests were performed (API 20E, API20 NE, API 20 Strep or API Staph; BioMérieux, Plainview, NY). The bacteria isolated were stored at $-70{ }^{\circ} \mathrm{C}$ until further analysis.

\subsection{Antimicrobial susceptibility testing}

Minimum inhibitory concentrations (MICs) of the three most frequently isolated uropathogens were determined according to the Clinical and Laboratory Standards Institute (CLSI) criteria (formerly the National Committee for Clinical Laboratory Standards) [14] using the microbroth dilution method with cation-adjusted Mueller-Hinton II broth (Becton, Dickinson and Company, Sparks, MD), an inoculum of $5 \times 10^{5} \mathrm{CFU} / \mathrm{mL}$ and an overnight incubation at $37^{\circ} \mathrm{C}$. MIC plates with freeze-dried antibiotics (NLDMCS1; MCS Diagnostics BV, Swalmen, The Netherlands) were delivered after quality testing by the manufacturer, with a shelf-life of ca. 1 year guaranteed. The following antimicrobial agents were tested (range in $\mathrm{mg} / \mathrm{L}$ ): amoxicillin $(0.06-128)$, amoxicillin/clavulanic acid (co-amoxiclav; 0.06-128), trimethoprim (0.03-64), cotrimoxazole (0.03-64), norfloxacin (0.03-64), ciprofloxacin (0.008-16) and nitrofurantoin (0.5-512). The reference strains Escherichia coli ATCC 35218 and ATCC 25922 were used as control strains. CLSI guidelines [14] for susceptibility breakpoints were used. 


\subsection{Statistical analysis}

For analysis of the culture results and the susceptibility percentages, the patients were divided in three age groups: 71-80, 81-90 and $>90$ years. Microbiological culture was considered the gold standard for the diagnosis of a UTI and was used to calculate the positive predictive value (PPV), negative predictive value, sensitivity and specificity of the nitrite and/or leukocyte esterase tests.

The statistical program SPSS 11.0 for Windows was used for statistical analysis. The Mann-Whitney U-test was performed to detect significant differences in prescribed therapy, isolated uropathogens and antibiotic susceptibility of the three most frequently isolated uropathogens. A Pvalue $<0.05$ was considered significant.

\section{RESULTS}

\subsection{Usual care of the GP}

In total, 917 female patients were included in the study, with a mean age of 80.1 years (range 71-104 years).

The performance characteristics of the diagnostic tests performed by the GPs are shown in Table 1. The nitrite test alone showed a high PPV (99\%) and a high specificity (97\%). For urine samples with a negative nitrite test, the leukocyte esterase test showed a PPV of $83 \%$ and a sensitivity of $78 \%$.

\section{[ TABLE 1 ]}

Ninety percent of the patients with a nitrite-positive urine sample were prescribed antibiotics by their GP (Table 2). Sixty-four percent of the patients whose urine samples showed a negative nitrite test and a positive leukocyte esterase test also received an antibiotic prescription. Of the patients with a urine sample that was negative by both the nitrite and leukocyte esterase tests, $27 \%$ received antibiotic therapy.

\section{[ TABLE 2 ]}

Nitrofurantoin was the antimicrobial agent most frequently prescribed in patients of all age groups (Fig. 1). The prescription rate ranged from $41 \%$ in the oldest to $51 \%$ in the youngest patients. Onequarter of the patients received fluoroquinolones, and trimethoprim was prescribed in $10-21 \%$ of all patients. The prescription rate of co-amoxiclav increased significantly from $2 \%$ to $8 \%$ with increasing age.

\section{[ FIGURE 1 ]}

Nitrofurantoin therapy was equally prescribed as a 3-, 5- and 7-day regimen (Fig. 2).

Fluoroquinolones were most frequently prescribed for 7 days and trimethoprim for 5 days in patients of all age groups.

\section{[ FIGURE 2 ]}

\subsection{Culture results}

In total, $88 \%(808 / 917)$ of the urine samples yielded a positive culture of $\geq 10^{3} \mathrm{CFU} / \mathrm{mL}$ (Table 3 ). Escherichia coli was the most frequently isolated uropathogen in all age groups (57-62\%). Proteus mirabilis was more frequently isolated in younger patients $(10 \%)$. Klebsiella pneumoniae was found significantly more often with increasing age, i.e. from $3 \%$ in patients aged $71-80$ years to $16 \%$ in patients aged $>90$ years. No Staphylococcus saprophyticus was isolated in this patient population.

\section{[ TABLE 3 ]}




\subsection{Susceptibility data}

Susceptibility percentages of the three most frequently isolated uropathogens per age group are shown in Table 4. The lowest susceptibility percentages for the three microorganisms were found for amoxicillin, trimethoprim and cotrimoxazole. Klebsiella pneumoniae showed the lowest susceptibility to nitrofurantoin (53-78\%; P. mirabilis excluded).

\section{[ TABLE 4 ]}

The MIC distributions for the three most frequently isolated uropathogens (Table 5) showed a bimodal curve for trimethoprim and co-trimoxazole. For the three microorganisms tested, the first peak was found at the susceptible side of the distribution. The distribution curves of E. coli and P. mirabilis showed a second peak at the highest concentration of both agents tested, whereas for K. pneumoniae this peak was situated at the breakpoint concentration for both agents. Furthermore, the peak of the distribution curves of norfloxacin and nitrofurantoin for K. pneumoniae were found at one and two concentration steps higher, respectively, compared with both other microorganisms.

\section{[ TABLE 5 ]}

\section{DISCUSSION}

To the best of our knowledge, this study is the first community-based study of acute uncomplicated UTIs in more than 900 non-institutionalised elderly females over the age of 70 years in The Netherlands.

The diagnosis of a UTI in elderly female patients is not always straightforward. In younger female patients it is known that the nitrite and leukocyte esterase tests have a high PPV for infection [8-10]. The performance characteristics of the nitrite and leukocyte esterase dipstick tests showed that in this elderly female population a positive nitrite test result also predicted an infection with a very high specificity. Furthermore, when the nitrite test was negative, a positive leukocyte esterase test still predicted a UTI in $83 \%$ of the urine samples. The sensitivity of this test was also relatively high (78\%). Therefore, both tests have been proven to predict a UTI accurately in the elderly noninstitutionalised female population. However, the combination of both diagnostic tests resulted in a false-negative test result in $17 \%$ of the urine samples, which might be explained by contaminating microorganisms, as obtaining a clean-catch urine specimen in the elderly is difficult [7]. Furthermore, bacteria need $\geq 4 \mathrm{~h}$ for the production of nitrite from nitrate at a reliable level, thus the nitrite dipstick test preferably requires first morning urine [10]. If the time span is too short for this conversion, falsenegative test results will be obtained. Furthermore, the biochemical reaction detected by the nitrite test is associated with members of the Enterobacteriaceae family, but not with other uropathogens such as Pseudomonas spp. or enterococci [10]. Indeed, the nitrite-negative samples showed E. coli significantly less frequently $(\mathrm{P}=0.000)$, but Enterococcus faecalis $(\mathrm{P}=0.069)$, Acinetobacter spp. $(\mathrm{P}$ $=0.000)$ and other Gram-negatives $(\mathrm{P}=0.000)$ and Gram-positives $(\mathrm{P}=0.005)$ significantly more often compared with the samples having a positive nitrite test. Also, as the cut-off value for positive culture used in this study was $\geq 10^{3} \mathrm{CFU} / \mathrm{mL}$, it might be that the number of nitrite-producing bacteria present in the urine was too low to obtain a detectable level of nitrite. Indeed, the samples in this study that had a negative nitrite test result significantly more often showed a culture of $10^{3}-10^{5} \mathrm{CFU} / \mathrm{mL}$ compared with the samples with a positive nitrite test (data not shown; $\mathrm{P}=0.000$ ).

GPs were seen to rely on the outcome of the diagnostic tests for the prescription of antimicrobial therapy, as $90 \%$ of the patients with a nitrite-positive urine sample received antimicrobial therapy. For patients with a negative nitrite and positive leukocyte esterase urine sample, the prescription rate dropped to $64 \%$, and this dropped even further to $56 \%$ in patients for whom neither test was performed. Sixty-eight percent $(n=84)$ of the patients with a urine sample negative for both the nitrite and leukocyte esterase tests did not receive antimicrobial therapy. Bacteriological culture was negative $\left(<10^{3} \mathrm{CFU} / \mathrm{mL}\right)$ in $42 \%(35 / 84)$ of these patients, who were therefore correctly not treated with antimicrobials. UTIs in the 49 culture-positive patients were predominantly caused by uropathogens other than E. coli, and in 28 of these patients a 'low-count' UTI, i.e. the urine samples showed 
bacterial growth of $10^{3}-10^{5} \mathrm{CFU} / \mathrm{mL}$, was present. Both factors might have caused the negative nitrite and leukocyte esterase test results. Unfortunately, no data were available on the course of the current episode of UTI in these patients. Therefore, the question of whether these patients were correctly not treated remains unanswered.

Although nitrofurantoin was the most frequently prescribed antimicrobial in the three age groups, there was an inverse relationship between the prescription rate of this agent and increasing age of the patients. In contrast, the number of patients prescribed co-amoxiclav and fluoroquinolones increased significantly with increasing age. Similar results were described by Goettsch et al. [15]. The oldest patients in their study received a prescription of fluoroquinolones more often compared with the younger ones. There might be several reasons for GPs choosing another first-line agent than those recommended by The Netherlands College of General Practitioners [16], i.e. nitrofurantoin and trimethoprim, in this older patient population. First, nitrofurantoin treatment regimens have several adverse effects, especially in older women, including nausea and vomiting, and allergic, pulmonary, hepatic, neurological and haematological reactions [17]. In addition, elderly females are more likely to have experienced several UTIs in their lifetime and therefore may have received antimicrobial therapy more often in the past [2-4]. Therefore, it is to be expected that the GP considered the standard agents as no longer effective and preferred to use co-amoxiclav or fluoroquinolones. These agents are also prescribed for longer durations to be sure of full eradication of the causative uropathogen. However, no significant differences in the antibiotic resistance rates between the different age groups were observed.

The microbial aetiology of UTIs among noninstitutionalised elderly women differed from that of younger female adults (unpublished data), i.e. E. coli, still the most common isolated uropathogen, was detected proportionally less, but P. mirabilis, K. pneumoniae and other Gram-negatives were isolated more. Owing to the depletion of hormones in these elderly patients, the vaginal flora is no longer dominated by the presence of lactobacilli [5] and consequently it might be easier for uropathogens to enter and colonise the urinary tract. Furthermore, S. saprophyticus was not isolated in this elderly patient population.

The lowest susceptibility percentages for the three most frequently isolated uropathogens were found for amoxicillin and trimethoprim, agents very often used in general practice. However, E. coli isolated in this patient population showed higher susceptibility percentages for amoxicillin, cotrimoxazole, norfloxacin and nitrofurantoin compared with uropathogenic E. coli isolated from residents of two nursing homes in the south of The Netherlands [12]. The differences in antibiotic susceptibility between the isolates from GP patients and nursing home residents is very likely due to the presence of additional risk factors for the development of antibiotic resistance in the latter population, including antibiotic use, crowding, underlying pathology and the presence of invasive devices $[12,18]$.

In conclusion, as elderly community-dwelling females mostly present to their GP with vague clinical symptoms of a UTI, it is important for the GP to perform the nitrite and leukocyte esterase dipstick tests to confirm their diagnosis of a UTI. Consequently, the percentage of patients incorrectly untreated with antibiotics will decrease. Furthermore, actual data on the susceptibility of pathogens isolated from this group of patients is essential for making an empirical choice for the treatment of UTIs in elderly females. Therefore, as trimethoprim and co-trimoxazole susceptibility percentages for the three most frequently isolated uropathogens in this population (patients $>90$ years of age excluded as the group was too small for any conclusions to be drawn) ranged from $73 \%$ to $86 \%$, it might be advisable not to use these antibiotics as a first-choice agent for the treatment of acute uncomplicated UTIs in The Netherlands. As nitrofurantoin is known to cause several side effects in the elderly population, the use of co-amoxiclav for the treatment of acute uncomplicated UTIs might be preferred over the use of fluoroquinolones. Co-amoxiclav has been available much longer compared with the fluoroquinolones but resistance against this agent has remained stable over time (E. coli $\sim 5 \%$ ) in contrast to resistance to fluoroquinolones, which is rapidly emerging [15].

National surveillance studies generating data on the resistance percentages of the agents currently used for the treatment of UTIs in patients of different age groups should be continued in the future and should be made available to GPs for implementation into their daily practice. 
Nys S, Merode T van, Bartelds AI, Stobberingh EE.

Antibiotic treatment and resistance of unselected uropathogens in the elderly.

International Journal of Antimicrobial Agents: 27, 2006, nr. 3, p. 236-41.

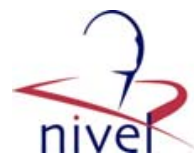

ACKNOWLEDGMENTS

This research was funded by the Dutch Foundation of the Working Party on Antibiotic Policy

(SWAB).We would like to thank all the General Practitioners of the Sentinel Station of The

Netherlands Institute for Health Services Research (NIVEL) for their participation in the study, and L.

Hoffman and S. Tonnaer for their technical assistance.

\section{TABLES AND FIGURES}

Table 1

Performance characteristics of the diagnostic tests performed by the General Practitioners

\begin{tabular}{|c|c|c|c|c|c|c|}
\hline \multirow[t]{2}{*}{ Diagnostic test result ${ }^{\mathrm{a}}$} & \multicolumn{2}{|l|}{ Urine culture } & \multirow[t]{2}{*}{ PPV } & \multirow[t]{2}{*}{ NPV } & \multirow[t]{2}{*}{ Sensitivity } & \multirow[t]{2}{*}{ Specificity } \\
\hline & $\geq 10^{3} \mathrm{CFU} / \mathrm{mL}$ & $<10^{3} \mathrm{CFU} / \mathrm{mL}$ & & & & \\
\hline \multicolumn{7}{|l|}{ Nitrite test in all patients } \\
\hline Positive & 409 & 3 & 99 & 22 & 52 & 97 \\
\hline Negative & 373 & 103 & & & & \\
\hline \multicolumn{7}{|c|}{ Leukocyte esterase test in cases of a negative nitrite test } \\
\hline Positive & 285 & 59 & 83 & 34 & 78 & 42 \\
\hline Negative & 82 & 42 & & & & \\
\hline
\end{tabular}

CFU, colony-forming units; PPV, positive predictive value; NPV, negative predictive value

a Samples with a negative nitrite and unknown leukocyte esterase test result or both unknown test results were excluded.

Table 4

Susceptibility percentages of the three most frequently isolated uropathogen

\begin{tabular}{|c|c|c|c|c|}
\hline \multirow{2}{*}{ Uropathogen } & \multirow[t]{2}{*}{ Antibiotic } & \multicolumn{3}{|c|}{ Age category (years) } \\
\hline & & $71-80$ & $81-90$ & $>90$ \\
\hline \multirow[t]{8}{*}{ Escherichia coli } & & $n=254$ & $n=137$ & $n=25$ \\
\hline & Amoxicillin & 69 & 74 & 80 \\
\hline & Co-amoxiclav & 95 & 96 & 96 \\
\hline & Trimethoprim & 76 & 80 & 84 \\
\hline & Co-trimoxazole & 79 & 83 & 84 \\
\hline & Norfloxacin & 93 & 98 & 96 \\
\hline & Ciprofloxacin & 93 & 98 & 96 \\
\hline & Nitrofurantoin & 98 & 97 & 100 \\
\hline \multirow[t]{7}{*}{ Proteus mirabilis } & & $n=30$ & $n=7$ & $n=1$ \\
\hline & Amoxicillin & 70 & 86 & 100 \\
\hline & Co-amoxiclav & 93 & 100 & 100 \\
\hline & Trimethoprim & 73 & 86 & 100 \\
\hline & Co-trimoxazole & 77 & 86 & 100 \\
\hline & Norfloxacin & 100 & 100 & 100 \\
\hline & Ciprofloxacin & 100 & 100 & 100 \\
\hline \multirow[t]{7}{*}{ Klebsiella pneumoniae } & & $n=8$ & $n=15$ & $n=9$ \\
\hline & Co-amoxiclav & 100 & 100 & 100 \\
\hline & Trimethoprim & 75 & 80 & 100 \\
\hline & Co-trimoxazole & 88 & 80 & 100 \\
\hline & Norfloxacin & 100 & 80 & 100 \\
\hline & Ciprofloxacin & 100 & 80 & 100 \\
\hline & Nitrofurantoin & 75 & 53 & 78 \\
\hline
\end{tabular}

Co-amoxiclav, amoxicillin/clavulanic acid.
Table 2

The number (rate) of prescriptions by the General Practitioners compared with the diagnostic test results

\begin{tabular}{llcc}
\hline Diagnostic test result & \multicolumn{3}{l}{ Therapy prescribed } \\
\cline { 2 - 4 } & Yes & No & Unknown \\
\hline Nitrite positive & $372(90 \%)$ & $30(7 \%)$ & $10(2 \%)$ \\
$\begin{array}{l}\text { Nitrite negative } \\
\text { Leukocyte esterase } \\
\text { positive }\end{array}$ & $221(64 \%)$ & $113(33 \%)$ & $10(3 \%)$ \\
$\begin{array}{c}\text { Leukocyte esterase } \\
\text { negative }\end{array}$ & $33(27 \%)$ & $84(68 \%)$ & $7(6 \%)$ \\
\begin{tabular}{l} 
Unknown \\
\hline
\end{tabular} & $9(56 \%)$ & $2(13 \%)$ & $5(31 \%)$ \\
\hline
\end{tabular}

Table 3

Percentage of uropathogens isolated for each age group

\begin{tabular}{|c|c|c|c|c|}
\hline \multirow[t]{2}{*}{ Microorganism } & \multicolumn{3}{|c|}{ Age category (years) } & \multirow[t]{2}{*}{ Total $(N=808)$} \\
\hline & $71-80(n=460)$ & $81-90(n=292)$ & $>90(n=56)$ & \\
\hline Escherichia coli & 62 & 60 & 57 & 61 \\
\hline Proteus mirabilis & 10 & 6 & 4 & 8 \\
\hline Klebsiella pneumoniae $^{*}$ & 3 & 8 & 16 & 6 \\
\hline Pseudomonas spp. & 5 & 4 & 7 & 5 \\
\hline Acinetobacter spp. & 4 & 2 & 2 & 3 \\
\hline Other Gram-negatives & 12 & 13 & 11 & 12 \\
\hline Enterococcus faecalis & 3 & 3 & 2 & 3 \\
\hline Other Gram-positives & 3 & 3 & 2 & 3 \\
\hline
\end{tabular}

* Significant difference between the youngest and both other age groups (Mann-Whitney $U$-test, $P<0.001$ ). 
Nys S, Merode T van, Bartelds AI, Stobberingh EE.

Antibiotic treatment and resistance of unselected uropathogens in the elderly.

International Journal of Antimicrobial Agents: 27, 2006, nr. 3, p. 236-41.

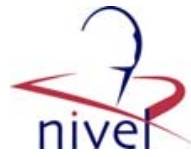

Table 5

Minimum inhibitory concentration (MIC) distributions of the three most frequently isolated uropathogens

\begin{tabular}{|c|c|c|c|c|c|c|c|c|c|c|c|c|c|c|c|c|}
\hline \multirow[t]{2}{*}{ Antimicrobial agent } & \multicolumn{14}{|c|}{ Percentage of isolates inhibited by the concentration $(\mathrm{mg} / \mathrm{L})$} & \multirow[t]{2}{*}{$\mathrm{MIC}_{50}$} & \multirow[t]{2}{*}{$\mathrm{MIC}_{90}$} \\
\hline & $\leq 0.03$ & 0.06 & 0.12 & 0.25 & 0.5 & 1 & 2 & 4 & 8 & 16 & 32 & 64 & 128 & $>128$ & & \\
\hline \multicolumn{17}{|c|}{ Escherichia coli $(n=416)$} \\
\hline Amoxicillin & & 0 & 0 & 0 & 0.2 & 3 & 14 & 44 & 10 & 1 & 0 & 1 & 1 & 27 & 4 & $>128$ \\
\hline Co-amoxiclav & & 0 & 0 & 0 & 0.2 & 3 & 21 & 43 & 19 & 9 & 3 & 1 & 0.2 & 0 & 4 & 16 \\
\hline Trimethoprim & 0.2 & 1 & 10 & 31 & 26 & 8 & 1 & 0.2 & 0 & 0.2 & 0 & 1 & 22 & & 0.5 & 128 \\
\hline Co-trimoxazole & 20 & 38 & 15 & 3 & 3 & 1 & 1 & 0 & 0 & 0 & 0 & 0.2 & 20 & & 0.06 & 128 \\
\hline Norfloxacin & 13 & 65 & 12 & 2 & 3 & 0.2 & 1 & 0.2 & 0.2 & 0.2 & 1 & 1 & 3 & & 0.06 & 0.12 \\
\hline Ciprofloxacin & 90 & 1 & 2 & 1 & 0.2 & 1 & 0 & 1 & 1 & 1 & 2 & & & & $\leq 0.03$ & $\leq 0.03$ \\
\hline Nitrofurantoin & & & & 0 & 0 & 0.2 & 1 & 4 & 26 & 58 & 7 & 1 & 2 & 0.4 & 16 & 32 \\
\hline \multicolumn{17}{|c|}{ Proteus mirabilis $(n=38)$} \\
\hline Amoxicillin & & 3 & 0 & 5 & 34 & 26 & 5 & 0 & 0 & 0 & 3 & 8 & 8 & 8 & 1 & 12 \\
\hline Co-amoxiclav & & 0 & 0 & 0 & 45 & 26 & 13 & 5 & 3 & 3 & 3 & 3 & 0 & 0 & 1 & 8 \\
\hline Trimethoprim & 0 & 0 & 3 & 0 & 3 & 26 & 16 & 18 & 11 & 0 & 0 & 0 & 24 & & 4 & 128 \\
\hline Co-trimoxazole & 3 & 32 & 40 & 0 & 0 & 0 & 5 & 3 & 0 & 0 & 3 & 3 & 13 & & 0.12 & 128 \\
\hline Norfloxacin & 13 & 68 & 11 & 0 & 5 & 3 & 0 & 0 & 0 & 0 & 0 & 0 & 0 & & 0.06 & 0.12 \\
\hline Ciprofloxacin & 84 & 8 & 8 & 0 & 0 & 0 & 0 & 0 & 0 & 0 & 0 & & & & $\leq 0.03$ & 0.06 \\
\hline \multicolumn{17}{|c|}{ Klebsiella pneumoniae $(n=32)$} \\
\hline Co-amoxiclav & & 0 & 0 & 0 & 3 & 13 & 44 & 28 & 6 & 6 & 0 & 0 & 0 & 0 & 2 & 8 \\
\hline Trimethoprim & 0 & 0 & 0 & 3 & 38 & 28 & 16 & 0 & 0 & 9 & 3 & 0 & 3 & & 1 & 16 \\
\hline Co-trimoxazole & 0 & 22 & 34 & 16 & 3 & 13 & 0 & 9 & 0 & 0 & 0 & 0 & 3 & & 0.12 & 4 \\
\hline Norfloxacin & 3 & 13 & 53 & 16 & 3 & 0 & 3 & 0 & 0 & 3 & 3 & 3 & 0 & & 0.12 & 2 \\
\hline Ciprofloxacin & 78 & 6 & 3 & 0 & 3 & 0 & 0 & 0 & 6 & 3 & 0 & & & & $\leq 0.03$ & 0.5 \\
\hline Nitrofurantoin & & & & 0 & 0 & 0 & 0 & 0 & 9 & 6 & 22 & 28 & 16 & 19 & 64 & $<128$ \\
\hline
\end{tabular}

Co-amoxiclav, amoxicillin/clavulanic acid.

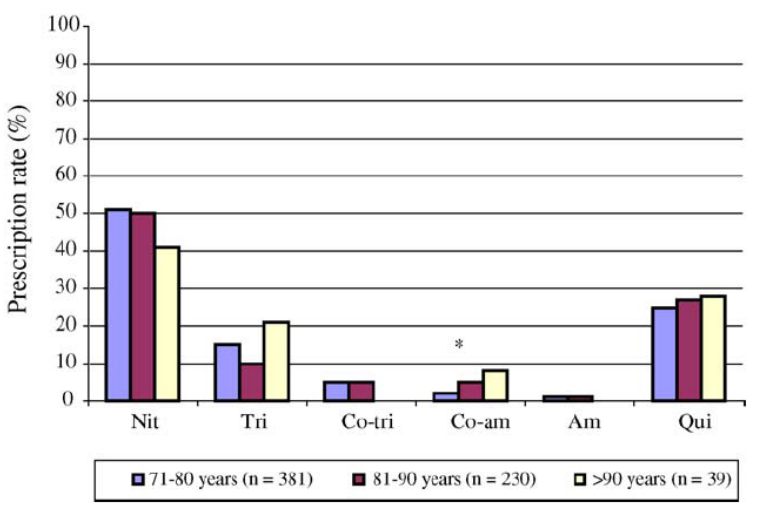

Fig. 1. Percentage of antimicrobial agents prescribed as therapy in patients with acute uncomplicated urinary tract infection by age category. Nit, nitrofurantoin; Tri, trimethoprim; Co-tri, co-trimoxazole; Co-am, amoxicillin/clavulanic acid; Am, amoxicillin; Qui, fluoroquinolones. *Significant difference between the youngest and both other age groups (Mann-Whitney $U$-test, $P<0.05$ )

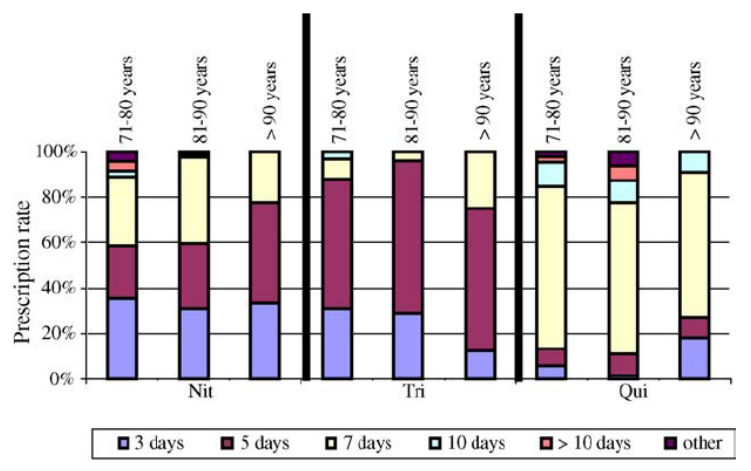

Fig. 2. Duration of antimicrobial therapy by age group for the three most commonly prescribed agents. Nit, nitrofurantoin; Tri, trimethoprim; Qui, fluoroquinolones.

\section{REFERENCES}

1. Nicolle LE. Urinary tract infection in the elderly. J Antimicrob Chemother 1994;33(Suppl. A):99-109.

2. Boscia JA, Kobasa WD, Knight RA, Abrutyn E, Levison ME, Kaye D. Epidemiology of bacteriuria in an elderly ambulatory population. Am J Med 1986;80:208-14.

3. Brown JS, Vittinghoff E, Kanaya AM, Agarwal SK, Hulley S, Foxman B. Urinary tract infections in postmenopausal women: effect of hormone therapy and risk factors. Obstet Gynecol 2001;98:1045-52.

4. van Geelen JM, van de Weijer PH, Arnolds HT. Urogenital symptoms and resulting discomfort in non-institutionalized Dutch women aged 50-75 years. Int Urogynecol J Pelvic Floor Dysfunct 2000;11:9-14.

5. Maloney C. Estrogen \& recurrent UTI in postmenopausal women. Am J Nurs 2002;102:44-52.

6. Nicolle LE. Urinary tract infection in long-term-care facility residents. Clin Infect Dis 2000;31:757-61.

7. Shortliffe LM, McCue JD. Urinary tract infection at the age extremes: pediatrics and geriatrics. Am J Med 2002;113(Suppl. 1A):55S-66S. 
Nys S, Merode T van, Bartelds AI, Stobberingh EE.

Antibiotic treatment and resistance of unselected uropathogens in the elderly.

International Journal of Antimicrobial Agents: 27, 2006, nr. 3, p. 236-41.

8. Christiaens T, De Meyere M, Derese A. Disappointing specificity of the leucocyte-esterase test for the diagnosis of urinary tract infection in general practice. Eur J Gen Pract 1998;4:144-8.

9. Verest LF, van Esch WM, van Ree JW, Stobberingh EE. Management of acute uncomplicated urinary tract infections in general practice in the south of The Netherlands. $\mathrm{Br} \mathrm{J}$ Gen Pract 2000;50:309-10.

10. Wilson ML, Gaido L. Laboratory diagnosis of urinary tract infections in adult patients. Clin Infect Dis 2004;38:1150-8.

11. Gupta K, Hooton TM, Roberts PL, Stamm WE. Patient-initiated treatment of uncomplicated recurrent urinary tract infections in young women. Ann Intern Med 2001;135:9-16.

12. Vromen M, van der Ven AJ, Knols A, Stobberingh EE. Antimicrobial resistance patterns in urinary isolates from nursing home residents. Fifteen years of data reviewed. J Antimicrob Chemother 1999;44:113-6.

13. Martin WJ, Birk RJ, Yu PK, Washington II JA. Identification of members of the family Enterobacteriaceae by the R-B system. Appl Microbiol 1970;20:880-3.

14. National Committee for Clinical Laboratory Standards. Methods for dilution antimicrobial susceptibility tests for bacteria that grow aerobically. 5th ed: Approved Standard M7-A5. Villanova, PA: NCCLS; 2002.

15. Goettsch W, van Pelt W, Nagelkerke N, et al. Increasing resistance to fluoroquinolones in Escherichia coli from urinary tract infections in The Netherlands. J Antimicrob Chemother 2000;46:223-8.

16. Wiersma TJ, Timmermans AE. Summary of the 'Urinary tract infections' guideline (first revision) of the Dutch College of General Practitioners. Ned Tijdschr Geneeskd 2001;145:735-9 [in Dutch].

17. Kunin $\mathrm{CM}$. Inappropriate medication use in older adults: does nitrofurantoin belong on the list for the reasons stated? Arch Intern Med 2004;164:1701.

18. Nicolle LE, Strausbaugh LJ, Garibaldi RA. Infections and antibiotic resistance in nursing homes. Clin Microbiol Rev 1996;9:1-17. 